\title{
Comparing Surgical Complications of Donors and Recipients in Retroperitoneoscopic versus Mini-Incision Donor Nephrectomy: A Single-Center Experience
}

\author{
Martina Koch, ${ }^{1}$ Alexander Bachmann, ${ }^{2}$ and Bjoern Nashan ${ }^{1}$ \\ ${ }^{1}$ Department of Hepatobiliary and Transplant Surgery, University Medical Centre, Hamburg-Eppendorf, \\ Martinistraße 52, 20246 Hamburg, Germany \\ ${ }^{2}$ Department of Urology, University Hospital Basel, Petersgraben 4, 4031 Basel, Switzerland
}

Correspondence should be addressed to Martina Koch; martina.koch@uke.de

Received 5 November 2012; Accepted 23 December 2012

Academic Editors: A. E. El-Agroudy, A. Rydzewski, and M. Veroux

Copyright (C) 2013 Martina Koch et al. This is an open access article distributed under the Creative Commons Attribution License, which permits unrestricted use, distribution, and reproduction in any medium, provided the original work is properly cited.

\begin{abstract}
Anterior vertical mini-incision donor nephrectomy (MIDN) has been used as the standard retrieval procedure in our center. Though the MIDN approach was tolerated very well with low complication rates, there were especially cosmetic reasons, to consider a change of procedure. Hence we switched to a total retroperitoneoscopic donor nephrectomy (RPDN) in 2011. We compared the outcome and surgical complications of donors and recipients of the first 30 RPDNs performed with 30 consecutive MIDN procedures. In both techniques, right and left nephrectomies were carried out. After a very short learning curve, the mean RPDN operation times were shorter compared to the MIDN (109 versus $171 \mathrm{~min}, P<0.01$ ) and donors were discharged earlier. No major complications occurred in the RPDN group and complications were less frequent compared to MIDN (17\% versus $40 \%)$. The renal function in the recipients was equivalent in both groups at the time of discharge and after one year. We conclude that RPDN is easy to learn for a surgical team experienced in open retroperitoneal donor nephrectomy. The change of the retrieval technique is safe for the donor and the recipient regarding surgical complications and recipients' renal function. Donors benefit from RPDN due to earlier hospital discharge and faster recovery.
\end{abstract}

\section{Introduction}

Living kidney donation is one of the most elective procedures in surgery requiring optimal care and technique. Beside providing a maximum of safety and comfort for the donor, short and long-term functional results of the donated kidney are of importance. Since the introduction of laparoscopic techniques into the donation procedure, various efforts were made to minimize the surgical burden to the donor.

Anterior vertical mini-incision donor nephrectomy (MIDN) is a minimal invasive approach compared to the standard flank incision $[1,2]$. Like the flank incision, it is a total retroperitoneal approach avoiding intra-abdominal alterations. In the past years, laparoscopic donation became more popular due to less postoperative pain and faster recovery of the donor compared to flank incision. Despite these benefits, there are concerns regarding intraabdominal alterations, such as adhesions of the intestine or injury of the diaphragm. These can result from mobilization of the colon flexure which is needed to provide access to the kidneys. For laparoscopic access with or without hand assistance long operation times $(>3 \mathrm{~h})$, the need for an experienced surgeon (long learning curve) and the potential life-threatening complications like splenic injuries have been described [3-5].

The retroperitoneoscopic technique first described by Yang et al. [6] combines the benefits of small incisions for the donor and the lack of complications of a transabdominal approach $[7,8]$.

In our center, donor nephrectomies were routinely performed in a retroperitoneal approach to avoid any possible intraabdominal complications. From 2007 to 2011, we used the anterior vertical MIDN as a standard technique and switched in the winter of 2011 to a retroperitoneoscopic 
approach following consultation and training at the University in Basel. Here we report the results of a single-center experience in the first consecutive 30 RPDN compared to 30 MIDN with special emphasis on complications in the donor and the recipient possibly related to the different retrieval techniques.

\section{Methods and Patients}

We compared the 30 anterior vertical MIDNs last performed before the switch to RPDN for the first 30 retroperitoneoscopic donor nephrectomies performed in 2011 and 2012 at the University Medical Center Hamburg Eppendorf. All patients received a standard preoperative evaluation procedure (including nephrological, surgical, and psychological examination) before being accepted as donors according to the German transplantation law. Medical examinations included magnetic resonance angiography (MRI) to evaluate anatomy of renal arteries and renal scintigraphy to evaluate renal function. The decision algorithm for choice of side for the nephrectomy was based on (1) standard anatomy of the kidney, assessed by MRI, and (2) functional size assessed by scintigraphy. Preferably, a kidney with a singular artery was selected, but multiple arteries were no exclusion criteria for donation. The algorithm of side decision was not changed during the study.

2.1. Surgical Procedure. MIDN was carried out as described before [1] with a $7-10 \mathrm{~cm}$ vertical pararectal incision and a total extraperitoneal approach. The retroperitoneoscopic technique described by Bachmann et al. [9-11] was applied with some modifications; briefly three $12 \mathrm{~mm}$ trocars were placed in a typical right- or left-sided overextended flank position. Starting with the herloon hernia balloon (Braun, Melsungen, Germany), a retroperitoneal space from an incision $1 \mathrm{~cm}$ below the 12 th rib and above the horizontal lateral line of the translucent erector trunci muscle was fashioned. The optical trocar was placed and a pressure of $12 \mathrm{mmHg}$ was used to create a sufficient retroperitoneal working space. Two other $12 \mathrm{~mm}$ trocars were placed above the hip and in the lower abdomen. Ureter and renal artery were identified and vessels were dissected using an ultracision harmonic knife (Ethicon, Germany). Prior to nephrectomy the medial trocar was extracted, and a gridiron incision of $6-7 \mathrm{~cm}$ (Figure 1) was used to allow one hand of the surgeon to assist the nephrectomy and to salvage the kidney. No hand port device was used. The ureter was clipped and dissected using Hemo-lok (Weck Closure Systems, Research Triangle Park, USA) clips and the renal vessels were transected with a multifire Endo TA30 (Autosuture, USA).

The organ implantation into the recipient was started immediately after organ retrieval in the same operation room and in most cases was performed by the same team.

2.2. Data Collection. All donors were monitored on the transplant unit and discharged when subjective well-being was sufficient. Pain medication was applied in a fixed schedule according to clinical standards with piritramid, and

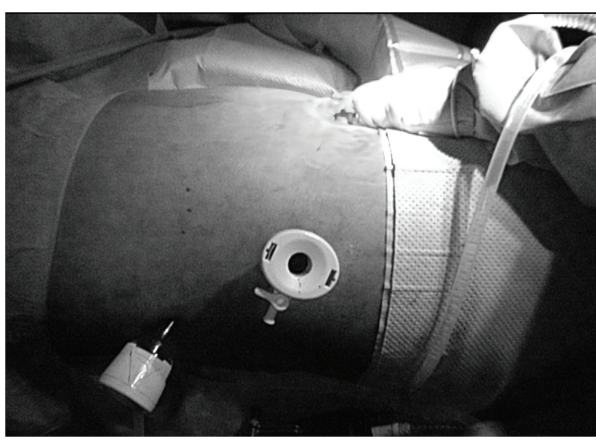

FIGURE 1: This figure shows the position of the hand of the surgeon passing the gridiron incision to assist dissection of the kidney vessels and two remaining $12 \mathrm{~mm}$ trocars.

paracetamol (acetaminophen) for at least 24 hours and thereafter with a combination of codeine/paracetamol.

All donors attended outpatient clinics at our center at least once after discharge and were informed to present themselves again in case of any problems associated with the donation. One year after donation, all patients were reevaluated by a nephrologist.

2.3. Statistics. Statistical analyses were performed using the two-sided Student's $t$-test (Microsoft Excel, Office 2003 for windows) and the Fisher's exact test using RGUI, R version 2.12.1, by the R Foundation for Statistical Computing 2010.

\section{Results}

There were no differences in donor and recipient demographics and surgical anatomy between both patient populations (Tables 1 and 2). More right-sided nephrectomies were performed in the MIDN group, an effect we would attribute to the small number of patients, since the selection algorithm of the donor side was not changed. In the RPDN group, more $\mathrm{ABO}$ incompatible transplants were carried out.

Skin to skin time was significantly shorter in patients with RPDN (mean 109 (70-237) min) compared to MIDN (mean 171 (112-228) $\mathrm{min}$ ). There was no difference in the operation time of a right (mean $167 \mathrm{~min}$ ) or left-sided nephrectomy (mean $179 \mathrm{~min}$ ) with MIDN, while the operation time of the right sided RPDN tended to be shorter with $98 \mathrm{~min}$. compared to $120 \mathrm{~min}$ on the left side. Neither number of arteries nor BMI had an impact on the skin to skin time.

The learning curve for the RPDN was short. Following the first donation, skin to skin time decreased significantly and was constantly below $2 \mathrm{~h}$ after 15 operations (Figure 2).

3.1. Outcome of the Donor. The followup of donors and recipients was 7-19 months in the RPDN group and 18-33 months in the MIDN group.

None of the donors died; no conversion to open surgery or surgical revision was necessary in any of the donors. There was no significant blood loss in any of the donors.

In the MIDN group, 12 of 30 patients developed complications. There were two major complications in one patient. 
TABLE 1: Donor demographics and outcome.

\begin{tabular}{lccc}
\hline & MIDN & RPDN & \\
\hline Number of patients & 30 & 30 & - \\
Male/female & $11 / 19$ & $9 / 21$ & n.s. \\
Age (years; mean and range) & $53(27-72)$ & $54(28-78)$ & n.s. \\
BMI & $27(21-34)$ & $25(20-35)$ & $10 / 20$ \\
Side of donation (right/left) & $21 / 9$ & $<0.01$ \\
Patient with more than 1 renal artery & $7(3$ previously unknown) \\
Mean operation time (min) & 171 & 109 & none \\
Blood transfusions & None & None & - \\
Conversion to open surgery/revision & None & $4,9^{*}$ & - \\
Donor hospitalization (days after OP) & 6.5 & $<0.01$ \\
${ }^{*}$ One patient was required to stay with the recipient (mental disabled) for 13 days and was excluded from the analysis.
\end{tabular}

TABLE 2: Recipient demographics and outcome.

\begin{tabular}{lccc}
\hline & MIDN & RPDN & $P$ \\
\hline Number of patients & 30 & 30 & - \\
\hline Related to the donor & Demographics & \\
ABOi transplants & $20 / 30$ & $15 / 30$ & n.s. \\
Age (years; mean) & 4 & 8 & n.s. \\
\hline \multicolumn{4}{c}{ Outcome } \\
\hline Renal vessel anastomosis time (min) & 35 & 28 & $<0.01$ \\
Renal function at time of discharge $\%$ & $100 \%$ & $93 \%$ & n.s. \\
S-creatinine at time of discharge (mg/dL) & 1.5 & 1.5 & n.s. \\
\hline
\end{tabular}

TABLE 3: Complications of the donor.

\begin{tabular}{lcc}
\hline & MIDN & RPDN \\
\hline Patients with at least one complication & $12 / 30(40 \%)$ & $5 / 30(17 \%)$ \\
UTI & 3 & 1 \\
Wound-healing problems & & \\
$\begin{array}{l}\text { superficial infection, prolonged } \\
\text { secretion) }\end{array}$ & 1 & 1 \\
Optical complains about the scar & 2 & 0 \\
Persisting pain & 3 & 2 \\
Lung embolism & 1 & 0 \\
Others & 3 & 1 \\
\hline
\end{tabular}

The 71-year-old donor developed lung embolism with the need of anticoagulation after donation and suffered from a small bowel perforation one month after donation. It is unknown if this event was related to the kidney donation.

Minor complications were urinary tract infection (UTI) in 3/30 patients and one pneumothorax treated conservatively. Persistent pain or esthetic complains about the scar were reported by $5 / 30$ patients. One patient had a resection of a vocal fold polyp after donation, which might have been related to the anaesthesia.

Following RPDN, only 5 of 30 patients (17\%) developed minor complications possibly associated with the donation. This was not statistically significant different from the MIDN

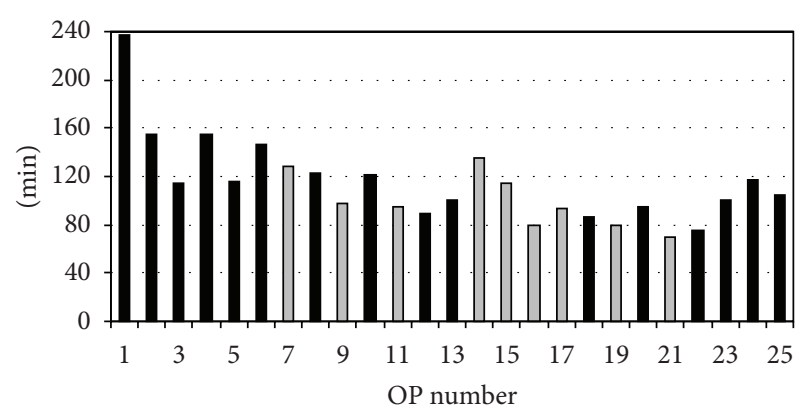

FIGURE 2: Skin to skin times of the first 25 RPDN performed. In black: left-sided nephrectomy, grey: right-sided nephrectomy.

group. Table 3 provides information about the type of complications in both groups.

Donors were discharged 6.5 [3-12] days after MIDN and 4.9 [3-13] days after RPDN, which was significantly different $(P<0.001$, Table 1$)$. None of the donors was forced to leave hospital before his or her subjective well-being. One donor in each group remained an inpatient for more than 10 days in order to provide psychosocial and mutual support for the recipient. In both cases, couples above the age of 70 were involved. One mother donated for her mentally retarded daughter and accompanied her during the whole hospital stay. This time was not counted for statistics.

3.2. Outcome of the Recipients. Although we did not measure the length of the renal artery or vein, there was no obvious difference regarding the quality and length of the vessels or the ureter with the open or retroperitoneoscopic approach. The mean time of vessel anastomosis was slightly shorter in the RPDN group ( $28 \mathrm{~min}$ ) compared to the MIDN ( $35 \mathrm{~min}$ ) suggesting that vessel anastomosis was not complicated by the RPDN technique. There was no renal artery or vein thrombosis in any of the recipients.

Other complications possibly associated with the retrieval technique such as ureter complications and lymphoceles were rare. $4 / 60$ patients $(6.7 \%)$ had minor ureter complications all treated successfully with a double J stent. In the MIDN group, one patient suffered from urinary leakage, which was 
treated with a double J stent and a suprapubic catheter, and one patient experienced ureter stenosis. One lymphocele occurred. In the RPDN group, one recipient was treated with a double J ureter stent and a suprapubic catheter due to a lesion of the renal pelvis, one with a double J stent for stenosis. All patients recovered without the need for operative revision.

$97 \%(58 / 60)$ of patients had a functioning graft at time of discharge. Two grafts in the RPDN group did not gain function: one patient lost his 72-year-old graft a due to an intima dissection and embolism of the intima flap. One highly sensitized patient with donor specific antibodies rejected the graft.

In both groups, the mean S-creatinine at the time of discharge was $1.5 \mathrm{mg} / \mathrm{dL}$.

1 year data are available in $26 / 30$ patient in the MIDN group with a graft survival of $25 / 26$ (96\%) and a mean screatinine of $1.4 \mathrm{mg} / \mathrm{dL}$. In the RPDN group 1 year follow up is completed in $18 / 30$ patients. All grafts were functioning with s-creatinine of $1.7 \mathrm{mg} / \mathrm{dL}$ which was not statistically significant from the MIDN group.

\section{Discussion}

Living donor kidney transplantation is the best medical option for patients on dialysis to reduce the waiting time for an organ of a deceased donor and to optimize the long-term outcome $[12,13]$. Nevertheless, safety of the donor is our main concern in a living organ donation.

Open lumbar flank incision with a retroperitoneal approach to the kidney has been a standard technique for the living donation for decades $[14,15]$. It is safe regarding the quality of the donated kidney and relatively easy to perform due to an optimal access in terms of space. The disadvantages of this technique are large scars, relatively long immobilization of the donor the and the risk of muscle relaxation of the flank in patients. To minimize these side effects minimal invasive open retroperitoneal approaches like the vertical miniincision have been established with excellent results $[16,17]$. Due to the limited access to the kidney, an experienced surgeon is needed for this operation. In this case, the operation time is comparable to the open approach $[1,2]$. Furthermore in the last 15-20 years, laparoscopic and retroperitoneoscopic approaches have been established. Regardless of the concerns about a higher rate of intraabdominal complications with a transabdominal approach and a longer operation time in most studies, the laparoscopic nephrectomy is more often reported compared to the retroperitoneoscopic approach. Studies comparing the retroperitoneoscopic and laparoscopic access are rare since most of the surgeons prefer one of the techniques. A study of Buell et al. compared a laparoscopic and retroperitoneoscopic approach for the right-sided nephrectomy in two different clinics in Ohio and stated that both techniques were safe with a longer operation time in the laparoscopic approach [18]. Ruszat et al. compared laparoscopic, open, and retroperitoneoscopic nephrectomies in Basel, Switzerland, pointing out a shorter operation time and faster learning curve for the retroperitoneoscopic nephrectomy [19]. These data and training in the Department of Urology in Basel, Switzerland encouraged us to reevaluate our centre policy of MIDN for living kidney donation.

Until winter 2011, we performed the MIDN, which was safe without any major complications in the donors as described earlier [1]. Some patients complained of prolonged pain (which might explain the UTIs due to late mobilisation keeping the urinary catheter in place for more than 1 day) and complications with the scare. From the surgical point of view, MIDN is complex especially in obese donors or donors with previous surgery. Therefore we changed our technique to RPDN in winter of 2011.

No conversion to open surgery was necessary during the initial learning phase and renal vessels were always safely controlled by the surgeon's hand entering the retroperitoneal space after mobilisation of the kidney. No significant bleeding occurred. Operation times were shorter in RPDN compared to the MIDN from the second case on and patients benefited from immediate mobilisation and an earlier hospital discharge. No major complication occurred in the first 30 RPDN cases and minor complications were reduced compared to MIDN.

Compared to the large published series of (hand-assisted) laparoscopic nephrectomies, the RPDN operation time was clearly shorter $[4,19,20]$, demonstrating that RPDN is relatively easy to perform. The clear view onto renal vessels provides a safe procedure for left- and right-sided kidney donation. Shorter anastomosis times during transplantation and the fact that no vascular complications were observed indicate that there is no difference in the quality of the vessels compared to MIDN. For right donor nephrectomy especially no problems with, for instance a short vein were encountered. In our RPDN group, more right nephrectomies were performed by chance. Operation times were slightly shorter on the right side, but this is caused by the fact that the first 6 operations were done on the left side and operation times decreased thereafter. Therefore we postulate that RPDN is equally suitable for both right- and left-sided nephrectomies.

None of the grafts was lost due to surgical complications. The outcome of grafts retrieved by MIDN or RPDN was comparable at time of discharge and after 1 year.

We demonstrate that RPDN can be learned within a series of 15-20 procedures by a surgeon experienced in open retroperitoneal surgery. The relatively small series reported here might not include all possible complications after PRDN, but it shows that the change in the operation technique is safe for both the donor and the recipient and that the donor profits from the less invasive technique even during a learning curve.

\section{Conclusion}

We conclude that living kidney donors benefit from a RPDN procedure compared to the open MIDN approach in terms of hospitalization and mobilisation. It was safe for donors and recipients to start RPDN with a team experienced in a retroperitoneal retrieval technique. Even during the learning phase, there were shorter operation times and quicker recovery of the donors after the RPDN approach. 
For surgical teams willing to switch the donor operation from an open approach to a minimal invasive operation, the retroperitoneoscopic approach is easy to learn and feasible for both right- and left-sided nephrectomies.

\section{Abbreviations}

MIDN: Mini-incision donor nephrectomy

RPDN: Retroperitoneoscopic donor nephrectomies

UTI: Urinary tract infection.

\section{Acknowledgment}

The authors would like to thank Dr. Hansjoerg Thude for the help with statistical analysis and Dr. Anja Lehnhardt for the critical review of the paper. The authors do not have a direct financial relation with the commercial identity mentioned in the paper and do not have a conflict of interest.

\section{References}

[1] M. Neipp, S. Jackobs, T. Becker et al., "Living donor nephrectomy: flank incision versus anterior vertical mini-incision," Transplantation, vol. 78, no. 9, pp. 1356-1361, 2004.

[2] A. A. Schnitzbauer, M. Loss, M. Hornung et al., "Mini-incision for strictly retroperitoneal nephrectomy in living kidney donation vs flank incision," Nephrology Dialysis Transplantation, vol. 21, no. 10, pp. 2948-2952, 2006.

[3] J. Wadström, "Hand-assisted retroperitoneoscopic live donor nephrectomy: experience from the first 75 consecutive cases," Transplantation, vol. 80, no. 8, pp. 1060-1066, 2005.

[4] J. F. Buell, M. J. Hanaway, S. R. Potter et al., "Hand-assisted laparoscopic living-donor nephrectomy as an alternative to traditional laparoscopic living-donor nephrectomy," American Journal of Transplantation, vol. 2, no. 10, pp. 983-988, 2002.

[5] J. L. Flowers, S. Jacobs, E. Cho et al., "Comparison of open and laparoscopic live donor nephrectomy," Annals of Surgery, vol. 226, no. 4, pp. 483-490, 1997.

[6] S. C. Yang, D. H. Lee, K. H. Rha, and K. Park, "Retroperitoneoscopic living donor nephrectomy: two cases," Transplantation Proceedings, vol. 26, no. 4, p. 2409, 1994.

[7] C. S. Ng, S. C. Abreu, H. I. Abou El-Fettouh et al., "Right retroperitoneal versus left transperitoneal laparoscopic live donor nephrectomy," Urology, vol. 63, no. 5, pp. 857-861, 2004.

[8] J. Wadström and P. Lindström, "Hand-assisted retroperitoneoscopic living-donor nephrectomy: initial 10 cases," Transplantation, vol. 73, no. 11, pp. 1839-1841, 2002.

[9] A. Bachmann, M. Dickenmann, L. Gürke et al., "Retroperitoneoscopic living donor nephrectomy: a retrospective comparison to the open approach," Transplantation, vol. 78, no. 1, pp. 168-171, 2004.

[10] A. Bachmann, T. Wolff, R. Ruszat et al., "Retroperitoneoscopic donor nephrectomy: a retrospective, non-randomized comparison of early complications, donor and recipient outcome with the standard open approach," European Urology, vol. 48, no. 1, pp. 90-96, 2005.

[11] T. Sulser, L. Gürke, I. Langer et al., "Retroperitoneoscopic living-donor nephrectomy: first clinical experiences in 19 operations," Journal of Endourology, vol. 18, no. 3, pp. 257-262, 2004.
[12] C. L. Davis and F. L. Delmonico, "Living-donor kidney transplantation: a review of the current practices for the live donor," Journal of the American Society of Nephrology, vol. 16, no. 7, pp. 2098-2110, 2005.

[13] H. U. Meier-Kriesche and B. Kaplan, "Waiting time on dialysis as the strongest modifiable risk factor for renal transplant outcomes: a paired donor kidney analysis," Transplantation, vol. 74, no. 10, pp. 1377-1381, 2002.

[14] T. L. Marchioro, R. S. Brittain, G. Hermann, J. Holmes, W. R. Waddell, and T. E. Starzl, "Use of living donors for renal homotransplantation," Archives of Surgery, vol. 88, pp. 711-720, 1964.

[15] M. C. Ottelin, A. J. Bueschen, L. K. Lloyd, D. B. Joseph, A. G. Diethelm, and J. R. Burns, "Review of 333 living donor nephrectomies," Southern Medical Journal, vol. 87, no. 1, pp. 6164, 1994.

[16] S. Jackobs, T. Becker, R. Lück et al., "Quality of life following living donor nephrectomy comparing classical flank incision and anterior vertical mini-incision," World Journal of Urology, vol. 23, no. 5, pp. 343-348, 2005.

[17] A. A. Schnitzbauer, M. Hornung, U. Seidel et al., "Does miniincision donor nephrectomy improve quality of life in living kidney donors?" Clinical Transplantation, vol. 21, no. 2, pp. 235240, 2007.

[18] J. F. Buell, S. C. Abreu, M. J. Hanaway et al., "Right donor nephrectomy: a comparison of hand-assisted transperitoneal and retroperitoneal laparoscopic approaches," Transplantation, vol. 77, no. 4, pp. 521-525, 2004.

[19] R. Ruszat, T. Sulser, M. Dickenmann et al., "Retroperitoneoscopic donor nephrectomy: donor outcome and complication rate in comparison with three different techniques," World Journal of Urology, vol. 24, no. 1, pp. 113-117, 2006.

[20] M. Sawatzky, A. Altaf, J. Ellsmere et al., "Is right laparoscopic donor nephrectomy right?" Surgical Endoscopy, vol. 23, no. 6, pp. 1321-1325, 2009. 


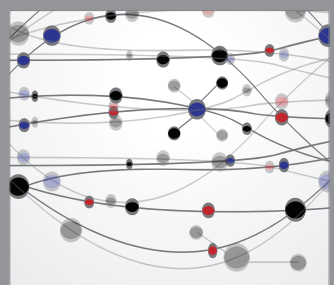

The Scientific World Journal
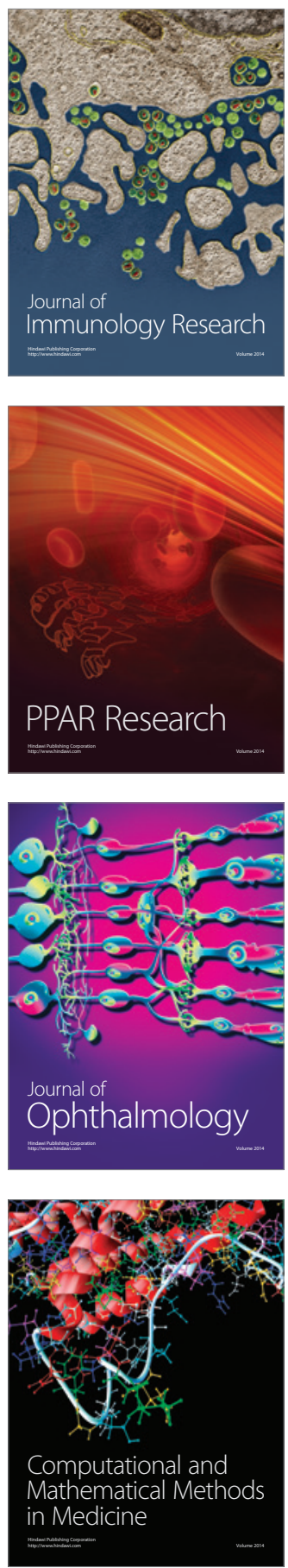

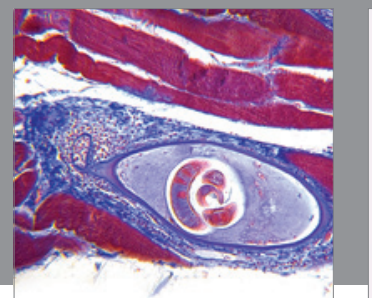

Gastroenterology

Research and Practice
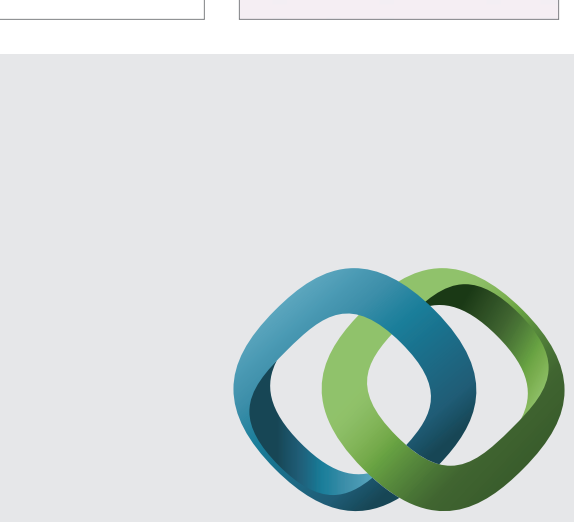

\section{Hindawi}

Submit your manuscripts at

http://www.hindawi.com
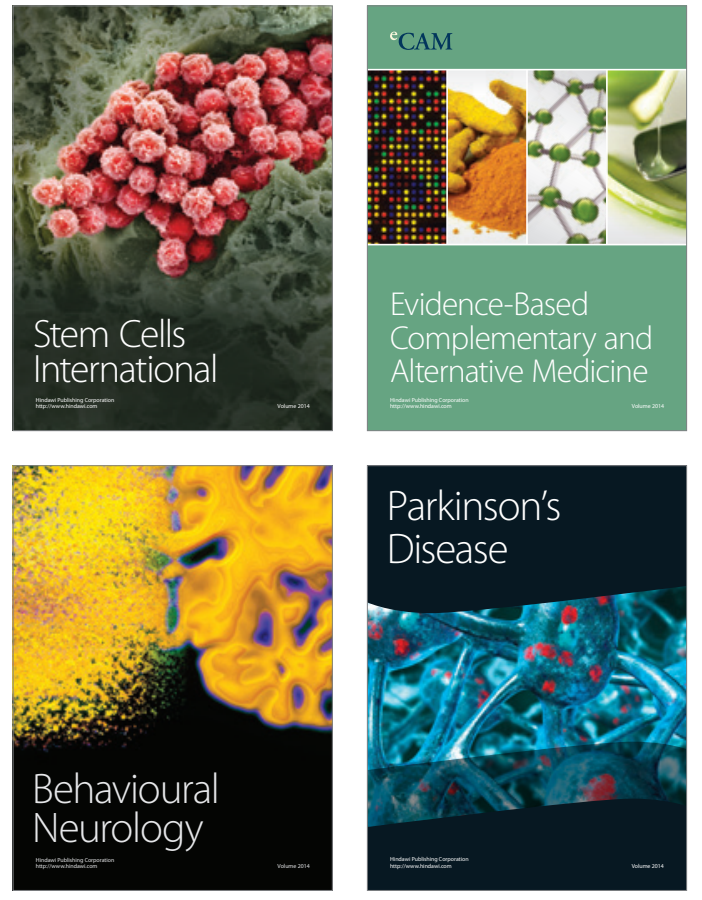
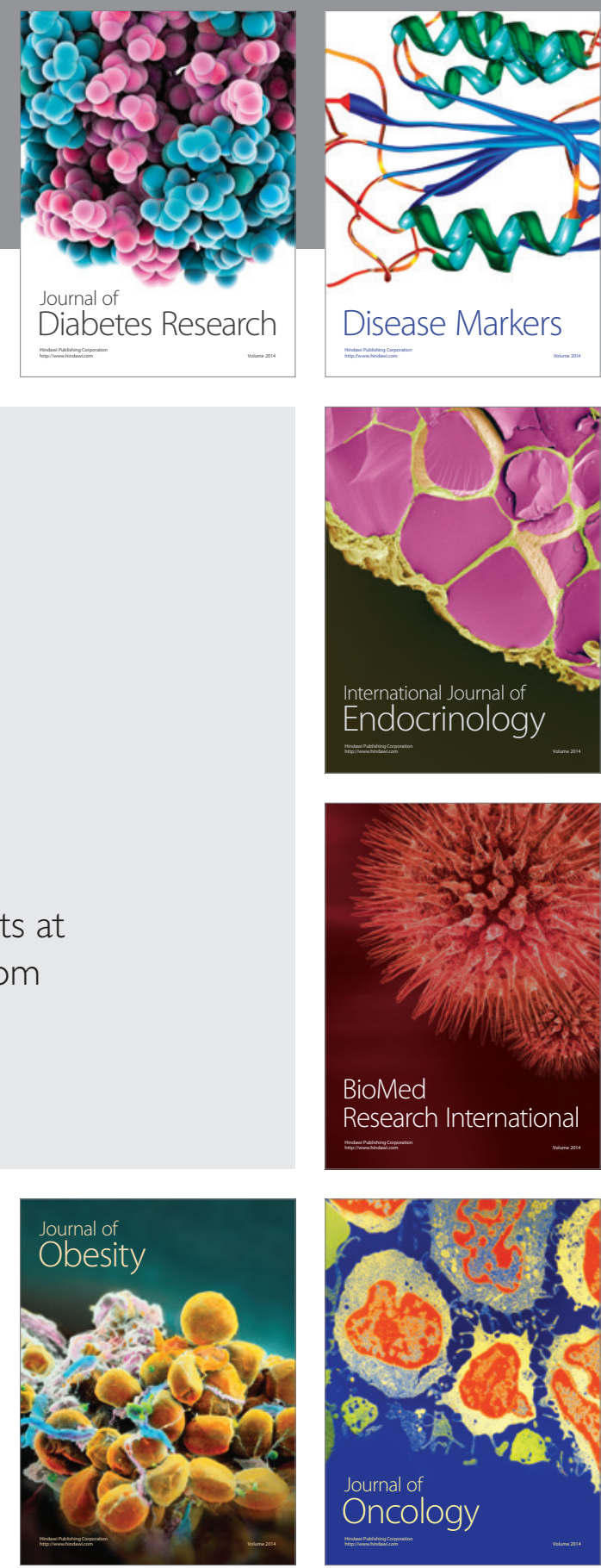

Disease Markers
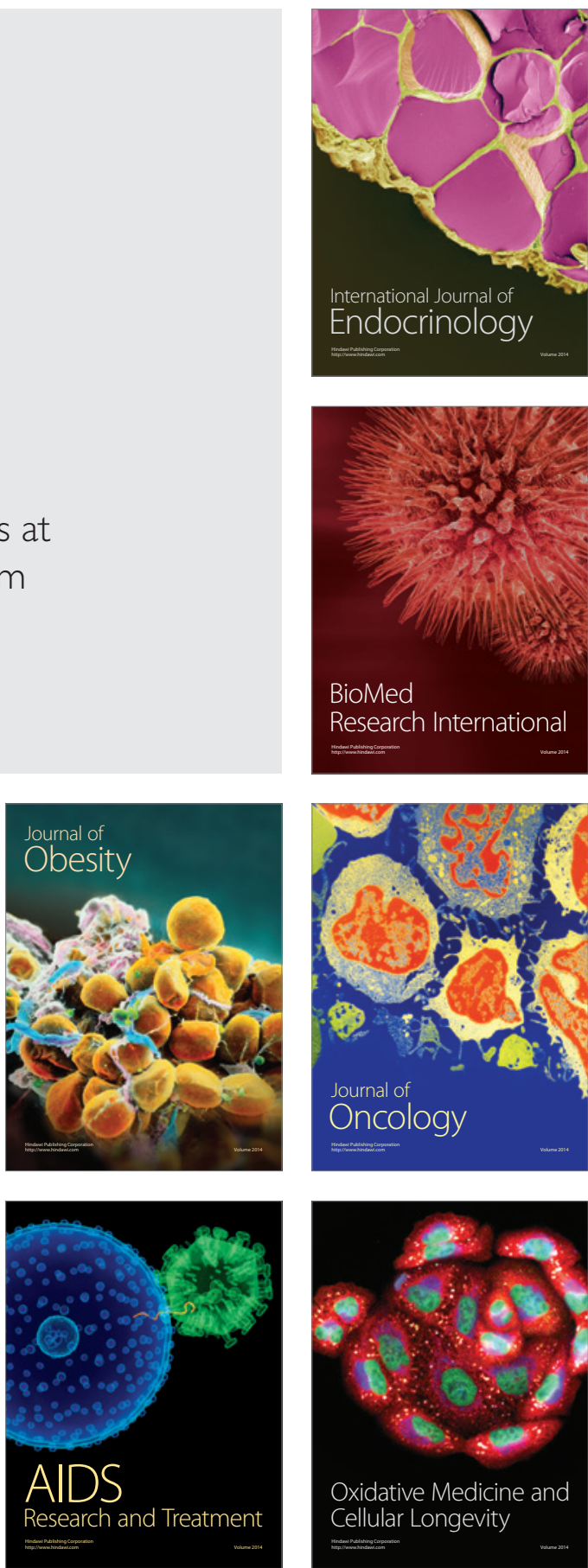